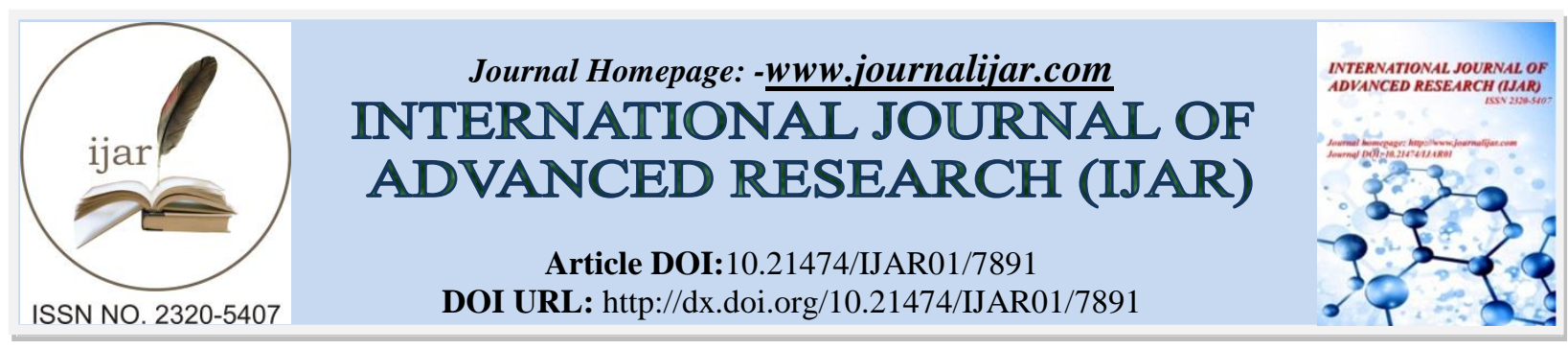

RESEARCH ARTICLE

\title{
USAGE OF INTRAMUSCULAR METHOTREXATE IN THE SUCCESSFUL MANAGEMENT OF CAESAREAN SCAR PREGNANCY: A RARE CASE REPORT.
}

\author{
Dr. ChandanKachru, Dr. CharuDuttArora, Dr. RaginiAggarwal and MehakBatra.
}

\section{Manuscript Info}

Manuscript History

Received: 11 August 2018

Final Accepted: 13 September 2018

Published: October 2018

Abstract

Copy Right, IJAR, 2018,. All rights reserved.

\section{Introduction:-}

Caesarean scar pregnancy is the rarest type of ectopic pregnancy, associated with a high risk of morbidities and complications, such as uterine disruption and extensive hemorrhage [1]. The incidence of scar pregnancies is increasing as witnessed, as reported by the number of cases reported in the recent few decades due to rising number of Caesarean sections being performed and widespread use of transvaginal scans which helps detect such pregnancies early [2]. Conservative treatment modalities such as dilatation and curettage, excision of trophoblastic tissues (laparotomy or laparoscopy), [3] local and/or systemic administration of methotrexate [4] , bilateral hypogastric artery ligation associated with trophoblastic evacuation, and selective uterine artery embolization combined with curettage and/or methotrexate administration[5].

In this paper, we will report a case of Caesarean scar pregnancy which was successfully treated by systemically administered methotrexate.

\section{Case report}

A 36-year-old woman presented with complaints of vaginal bleeding for three hours with around one and a half month of amenorrhea coupled with a positive urine pregnancy test. She had a history of two cesarean sections; the first one was performed 8 years ago while the second one was 4 years ago due to fetal distress.

On physical examination, vital signs were found to be stable. Per abdomen was soft with no scar tenderness while in bimanual examination, few clots were removed, os was closed and an enlarged uterus of approximately 6 weeks in size with no adnexal mass was palpable.

Transvaginal ultrasound revealed Single intra-uterine gestational sac, which exposed an embryo and yolks, noted in the lower upper part of uterine cavity overlying previous LSCS scar. Scar thickness was 7.2 mm, cardiac activity was absent and there was no fluid in the cul-de-sac. The serum level of the $\beta$-subunit of human chorionic gonadotrophin (ß-hCG) was $1129.7 \mathrm{mUI} / \mathrm{mL}$.

These findings were compatible with a caesarean scar pregnancy. A magnetic resonance imaging revealed borderline bulky uterus with the mild irregularity of endometrium in the lower part of uterus anteriorly with slight anterior breaking due to endometrial thickening extending along scar line and hemorrhagic content in lower part of endometrial cavity which too extended along the scar line. There was no extension of endometrial thickening up to 
serosal surface anteriorly with normal plane between anterior uterine wall and urinary bladder but a relative thinning of the anterior wall of the uterus at scar site.

The patient was counseled regarding her management options, and since she had two Caesarean sections in the past, she opted for the medical treatment.

She received the first dose (Day 0) of Methotrexate $(50 \mathrm{mg}: 1 \mathrm{mg} / \mathrm{Kg}$ of body weight) intramuscularly. The serum level of the $\beta$-subunit of human Chorionic Gonadotrophin at the 4th day was $188.79 \mathrm{mUI} / \mathrm{mL}$ requiring a second dose of methotrexate. Slight vaginal bleeding followed this second dose and the $\beta$-hCG levels on day 8 decreased to $42.11 \mathrm{mUI} / \mathrm{mL}$. Following this, the patient was discharged and followed up as an outpatient until B-hCG levels, at day 21 was $12.4 \mathrm{mUI} / \mathrm{mL}$.

\section{Discussion:-}

The true incidence and prevalence of Caesarean scar ectopic pregnancy are difficult to determine because of its rarity. Risk factors for an ectopic pregnancy to develop in a Caesarean scar are many, including former dilatation and curettage, previous placental pathology, manual removal of placenta, ectopic pregnancy, in vitro fertilization (IVF), two or more previous Caesarean sections, and other uterine surgery such as myomectomy, metroplasty, or hysteroscopy.

The genesis of this type of pregnancy may involve the implantation of the pregnancy into the myometrium via a microscopic tract, or perhaps a dehiscence in the previous uterine scar[6].

The most important investigation is ultrasonography with Doppler flow studies. The sonographic criteria for diagnosing a pregnancy in a scar are

1. an empty uterus;

2. an empty cervical canal;

3. a gestational sac located in the anterior part of the isthmic portion of the uterus, with a diminished myometrial layer between the bladder and the sac;

4. a discontinuity in the anterior wall of the uterus demonstrated on a sagittal view of the uterus (when the direction of the ultrasound beam runs through the amniotic sac). Additionally, vascular flow is demonstrated around the gestational mass.

The vial has suggested that there may be two different types of Caesarean scar pregnancies. The first type involves implantation of the gestational sac on the scar with progression towards either the cervix or the uterine cavity. In this type, the fetus may grow to viability but there is a risk of life-threatening hemorrhage from the implantation site. The second type involves a deep implantation in a Caesarean scar defect with possible rupture and bleeding during the first trimester of pregnancy. Vial comments that these differences may help to identify the most appropriate treatment.

In the hemodynamically stable patient, medical or surgical options for management may be considered. The primary objective is to eliminate the gestational sac and preserve the patient's fertility. Medical treatment usually consists of either systemic or local injection with methotrexate. Potassium chloride can be used for local injection. Previous case reports have suggested that it may be prudent to consider systemic injection of methotrexate rather than local injection.

The surgical approach includes radical and conservative procedures. The radical procedure consists in hysterectomy when the uterus is ruptured or if bleeding is uncontrollable. The conservative procedure includes (i) evacuation of the pregnancy and repair of the uterine defect by laparotomy or laparoscopy, (ii) dilatation and curettage and excision of trophoblastic tissues using laparotomy or laparoscopy, and (iii) bilateral hypogastric artery ligation associated with $\mathrm{D}$ and $\mathrm{C}$ under laparoscopic guidance.

Another treatment possibility is the uterine artery embolization (UAE), which is widely accepted as a conservative treatment in postpartum hemorrhage, in uterine fibroids, it is also considered as the best method to prevent massive bleeding during D and C for cervical pregnancy [7]. Although UAE seems to be promising in treating stable cases, it is not recommended as a primary line therapy.

In our case, since the patient was stable, she did not want to have a surgical procedure and since there were no facilities for UAE, we opted for medical treatment. 


\section{Conclusion:-}

In this study, we demonstrated that Caesarean scar pregnancy can be treated safely by systemic methotrexate injection. A hysteroscopy may be performed a few months after treatment to evaluate the Caesarean section scar. Decisions on treatment options should be dictated in part by gestational age, hCG levels, the presence of fetal cardiac activity, the desire of future fertility, and the experience and facilities available. Counseling patients with Caesarean scar pregnancy is not easy since there is no data about the optimum treatment. More reports are desirable to rationalize the treatment modalities for this condition.

\section{References:-}

1. A. Herman, Z. Weinraub, O. Avrech, R. Maymon, R. Ron-El, and Y. Bukovsky, "Follow up and outcome of isthmic pregnancy located in a previous caesarean section scar," British Journal of Obstetrics and Gynaecology, vol. 102, no. 10, pp. 839-841, 1995.

2. D. L. Fylstra, T. Pound-Chang, M. G. Miller, A. Cooper, and K. M. Miller, "Ectopic pregnancy within a cesarean delivery scar: a case report," American Journal of Obstetrics and Gynecology, vol. 187, no. 2, pp. 302304, 2002.

3. K.-M. Seow, W.-C. Cheng, J. Chuang, C. Lee, Y.-L. Tsai, and J.-L. Hwang, "Methotrexate for cesarean scar pregnancy after in vitro fertilization and embryo transfer: a case report," Journal of Reproductive Medicine for the Obstetrician and Gynecologist, vol. 45, no. 9, pp. 754-757, 2000.

4. M.-J. Yang and M.-H. Jeng, "Combination of transarterial embolization of uterine arteries and conservative surgical treatment for pregnancy in a cesarean section scar: a report of 3 cases," Journal of Reproductive Medicine for the Obstetrician and Gynecologist, vol. 48, no. 3, pp. 213-216, 2003.

5. F.-T. Kung, T.-L. Huang, C.-W. Chen, and Y.-F. Cheng, "Cesarean scar ectopic pregnancy," Fertility and Sterility, vol. 85, no. 5, pp. 1508-1509, 2006.

6. O. Graesslin, F. Dedecker Jr., C. Quereux, and R. Gabriel, "Conservative treatment of ectopic pregnancy in a cesarean scar," Obstetrics and Gynecology, vol. 105, no. 4, pp. 869-871, 2005.

7. J. Sugawara, M. Senoo, H. Chisaka, N. Yaegashi, and K. Okamura, "Successful conservative treatment of a cesarean scar pregnancy with uterine artery embolization," Tohoku Journal of Experimental Medicine, vol. 206, no. 3, pp. 261-265, 2005. 